\title{
Neuromuscular and Mobility Responses to a Vibration Session in Hypoxia in Multiple Sclerosis
}

\author{
Authors \\ Luis Andreu-Caravaca1, 2 D, Linda H. Chung ${ }^{3}$, Domingo Jesús Ramos-Campo ${ }^{4}$, Elena Marín-Cascales ${ }^{3}$, \\ Alberto Encarnación-Martínez ${ }^{5}$, Jacobo Á. Rubio-Arias ${ }^{6}$
}

\section{Affiliations}

1 International Chair of Sports Medicine, Universidad Católica San Antonio de Murcia, Murcia

2 Faculty of Sport, Universidad Católica San Antonio de Murcia, Murcia

3 UCAM Research Center for High Performance Sport, Universidad Católica San Antonio de Murcia, Murcia

4 Sport Science, Universidad Catolica San Antonio de Murcia, Murcia

5 Department of Physical Education and Sports, Research Group in Sport Biomechanics (GIBD), University of Valencia, Valencia

6 LFE Research Group, Department of Health and Human Performance, Universidad Politecnica de Madrid, Madrid

Key words

vibration training, neurological disorders, strength, muscle oxygenation, functional capacity

accepted $\quad 13.09 .2020$

published online 2020

\section{Bibliography}

Int J Sports Med

DOI 10.1055/a-1273-8304

ISSN $0172-4622$

(C) 2020. Thieme. All rights reserved.

Georg Thieme Verlag KG, Rüdigerstraße 14,

70469 Stuttgart, Germany

\author{
Correspondence \\ Luis Andreu-Caravaca \\ International Chair of Sports Medicine, Universidad Católica \\ San Antonio de Murcia \\ Campus de los Jerónimos \\ 30107 Murcia \\ Spain \\ Tel.: (+ 34) 9682788 00, Fax: (+34) 968278800 \\ landreu@ucam.edu
}

\section{ABSTRACT}

The aim of this study was to investigate the acute effects of vibration training (WBVT) under hypoxic and normoxic conditions on the voluntary rate of force development (RFD), balance and muscle oxygen saturation $\left(\mathrm{SMO}_{2}\right)$ in persons with Multiple Sclerosis (MS). 10 participants completed the study (30\% males, $44.4 \pm 7.7$ years, $164.3 \pm 8.9 \mathrm{~cm}, 65.2 \pm 11.1 \mathrm{~kg}, 2.5 \pm 1.3$ Expanded Disability Status Scale, $\left.24.1 \pm 4.0 \mathrm{~kg} \cdot \mathrm{m}^{-2} \mathrm{BMI}\right)$. Maximal force, RFD during isometric knee extension, static balance with eyes open and closed and sit-to-stand test were evaluated before and immediately after one session of WBVT (12 60-s bout of vibration; frequency $35 \mathrm{~Hz}$; amplitude $4 \mathrm{~mm}$; 1 -min rest intervals) under both normoxic and hypoxic conditions. In addition, $\mathrm{SMO}_{2}$ of the gastrocnemius lateralis was assessed during each condition. No changes were found in force, static balance and sit-to-stand test. Time-to-peak RFD increased in the left leg $(p=0.02)$ and tended to increase in the right leg $(p=0.06)$ after the hypoxic session. $\mathrm{SMO}_{2}$ resulted in significant increases from the initial to final intervals of the WBVT under both hypoxic and normoxic conditions $(\mathrm{p}<0.05)$. Increases in $\mathrm{SMO}_{2}$ during WBVT demonstrates muscle work that may contribute to the observed muscle adaptations in long-term WBVT programs without inducing decreases in neuromuscular activation, physical function and balance within a session.

\section{Introduction}

Multiple sclerosis (MS) is the one of the main causes of disability in both young and middle-aged adults [1], affecting approximately 1.3 million people worldwide [2]. MS symptoms include various motor and sensory dysfunctions, one of them being the deterioration of postural control [3] due to declines in muscle strength [4]. Muscle weakness [5], spasticity, and ataxia [6] are common physi- cal disabilities, and as a consequence of these impairments, persons with MS (pwMS) present limitations in daily life tasks, which can lead to poor quality of life [7].

Although medication can ameliorate MS symptoms to a certain degree [8], it has been well-demonstrated that physical exercise can serve as a complement to medical treatment and improve functional mobility and autonomy in pwMS [9]. Exercise has shown to 
inhibit demyelination in animal models [10] and increase the release of neurotrophic factor [11], suggesting that it provides neuroprotective adaptations. In addition, exercise has shown to improve muscular strength, balance, and aerobic capacity in pwMS [12-14]. One particular training protocol, whole-body vibration training (WBVT), has shown to be highly effective on the central nervous system by inducing rapid muscle contraction and relaxation due to the mechanical multidimensional oscillations of the vibratory platform. Spinal inhibition, decreases in H-reflex, and facilitation in corticospinal excitability have been observed with WBVT [15]. The decreased excitability of the H-reflex after WBVT indicates that there is some inhibition of la afferent pathways of the spinal circuits, thus facilitating central drive [16], which could improve motor control during balance tasks and muscle coordination during walking in patients with neurological disorders $[17,18]$. WBVT has demonstrated improvements in balance in both healthy individuals [19] and pwMS [20,21] via increases in maximal strength and voluntary rate of force development (RFD) of the lower limb muscle groups [22, 23].

WBVT is a favorable exercise modality for pwMS because of its direct effect on the neuromuscular component, and it generates a low amount of symptomatic fatigue [24, 25]. In addition, research that has examined the acute effects of a WBVT session found that variables such as the RFD or the peak force are increased after a bout of this training in healthy population [26]. However, it is not clear what the acute neuromuscular alterations are following one session of WBVT in pwMS.

Another type of modality that could benefit pwMS is hypoxic training. Because of its potential benefits, the use of hypoxia in strength training has aroused much interest in the last decade [27], as it has been shown to alter the intramuscular environment and, consequently, enhance force production [28]. Hypoxia induces a specific molecular response of the oxygen-sensing pathways [29] that increases oxygen transport and lactate production, generating ATP via anaerobic metabolism [30]. When compared to a normoxic condition, exercise in hypoxia has shown to improve muscle blood flow, activate compensatory vasodilation, increase baseline oxygen consumption [31] and have a greater reliance on anaerobic metabolism [32]. Furthermore, increased motor unit recruitment is also observed [33] due to the greater increase in skeletal muscle, capillarization, and vascular endothelial growth factor [34]. Currently, only one study by Andreu et al. [24] has investigated the acute effects of hypoxia during WBVT training and demonstrated that one WBVT session does not worsen strength or mobility in pwMS. However, it is not known how hypoxia may affect neuromuscular activation, RFD and balance following a session of WBVT. Therefore, the overall effects of hypoxia and WBVT could be beneficial for pwMS, since both training systems use moderate-tohigh intensity with low production of fatigue while enhancing neuromuscular activation, which is a variable closely related to many tasks of daily life [35].

Thus, the main objectives of this study were to: 1) examine the acute effect of WBVT on RFD, physical function, and balance in pwMS after one WBVT session, and 2) compare these effects between normoxic and hypoxic conditions. Our main hypothesis was that WBVT would acutely decrease RFD, force (F), sit-to-stand time, and static balance. Our secondary hypothesis was that WBVT in hy- poxia would further diminish the aforementioned variables due to the greater motor unit recruitment during the session.

\section{Materials and Methods}

\section{Design}

All aspects of this cross-over study design were performed the UCAM Research Center for High Performance Sport and UCAM Sport Center (Murcia, Spain). Initially, subjects were randomly assigned to a WBVT session either under normoxic $\left(\mathrm{WBVT}_{\text {norm; }}\right.$; $\mathrm{FiO}_{2}=20.9 \%$ ) or hypoxic $\left(\mathrm{WBVT}_{\text {hyp }} ; \mathrm{FiO}_{2}=15.0 \%\right.$ ) conditions. In the first visit, familiarization of all testing procedures and the characteristics of the training session (i. e., different vibration frequencies and amplitudes) were carried out. Following 1 week (visit 2), subjects returned at the same time of day to perform the assigned WBVT condition (normoxia or hypoxia). After a week of rest, subjects repeated the WBVT protocol but under the condition not yet performed (visit 3). Hypoxia was carried out in a normobaric chamber (CAT-430; Colorado Altitude Training, Louisville, CO, USA) that had $15 \%$ reduced oxygen content facilitated by the CAT- 12 generator (Colorado Altitude Training). Consort guidelines for randomized clinical trials were followed. This study received approval from the Science Ethics Committee of the Catholic University of Murcia, in accordance with the Declaration of Helsinki [36] and meets the ethical standards of the journal [37]. This project was enlisted in ClinicalTrials.gov (identifier: NCT03856801).

\section{Participants}

A total of 13 pwMS were recruited from the local MS association. Participants ( 5 males and 7 females, $42.3 \pm 9.6$ years old, $164.2 \pm$ $8.5 \mathrm{~cm}$ tall, $67.4 \pm 12.8 \mathrm{~kg}$ of body mass, $2.8 \pm 1.5$ Expanded Disability Status Scale (EDSS), and with a body mass index of $29.6 \pm 5.6 \mathrm{~kg} \cdot \mathrm{m}^{-2}$ ) had relapsing-remitting MS, had no need of walking aids, and were not performing in any physical training programs (i. e., resistance and or aerobic). The diagnosis of MS was previously determined by a board-certified neurologist using the McDonald criteria [38]. The inclusion criteria were if participants: (1) had mild or moderate disability with clinical mild spastic-ataxic gait disorder, and (2) were in the stable phase of the disease. PwMS were excluded if they: (1) had an EDSS > 6, (2) had suffered a relapse in the prior 12 months, (3) were on corticosteroid treatment in the preceding 2 months, and (4) were participating in a resistance training program in the past 6 months. A written and signed informed consent was given by all participants before commencing the study.

\section{WBVT procedure}

All WBVT sessions were performed on the Power Plate Pro5 (Power Plate International, London, UK). Participants were in an isometric squat position with the knee flexed at $30^{\circ}$ [25]. As recommended by a previous study [14], the vibration frequency and peak-to-peak amplitude were fixed at $35 \mathrm{~Hz}$ and $4 \mathrm{~mm}$, respectively. For safety, participants were allowed to grip lightly onto the rail of the platform. The protocol was comprised of 12 sets of 1 -min isometric squats. The participant remained on the platform and stood upright during the 1 -min rest periods between sets. The rating of perceived exertion scale (6-20) was provided continuously during the 
session. If the subject exceeded a score on the scale of 17 (very hard), the session was terminated.

\section{Testing procedure}

Prior to and following the WBVT session, tests were conducted in the laboratory by the same investigator. A standardized 5-min warm-up on a cycle ergometer (75W; Technogym, Gambettola, Italy) and a dynamic stretching sequence were performed. The same test order was used in both intervention visits. The primary outcomes of neuromuscular performance were voluntary RFD $\left(R_{\text {early }}=\right.$ RFD 0-50 ms; $R_{\text {late }}=$ RFD 0-200 ms; RFD max and RFDtimetopeak $)$ and Force $(F)\left(F_{0-50}, F_{0-200}\right.$, and $\left.F_{\text {Time-to-peak }}\right)$. The secondary outcomes were muscle oxygenation of gastrocnemius lateralis $\left(\mathrm{SMO}_{2}\right)$, and different variables of static balance (mean anterior/ posterior displacement = MAPD; mean medial/lateral displacement = MMLD; total sway displacement $=$ TSD, and sway area $=S A$ ) under two conditions: eyes open (EO) and eyes closed (EC).

\section{Muscle oxygen saturation}

Muscle oxygen saturation $\left(\mathrm{SMO}_{2}\right)$ of the gastrocnemius lateralis was continuously monitored during sessions using a near-infrared spectroscopy system, the Moxy 3-Sensor Bundle (Fortiori Design LLC, Hutchinson, MN, USA). The average $\mathrm{SMO}_{2}$ was calculated every 3 sets $\left(\mathrm{SMO}_{21-3}, \mathrm{SMO}_{24-6}, \mathrm{SMO}_{27-9}, \mathrm{SMO}_{210-12}\right)$ in both legs at the same time. Peripheral oxygen saturation (MD300C2; ChoiceMMed, Beijing, China) was also measured to ensure that the participants were in a hypoxic situation. The measurements were taken at the beginning (before set 1 ), in the middle (before set 6 ) and at the end (after set 12) of the training session.

\section{Neuromuscular testing: force and rate of force development}

Participants sat upright on an isokinetic dynamometer chair (Biodex Medical Systems, Shirley, NY, USA) with both the left and right legs flexed at $90^{\circ}$ and the ankle strapped to a customized apparatus that held a load cell (Model SML500; Interface Inc., Scottsdale, AZ, USA). Participants carried out 3 trials of maximal voluntary isometric contraction (MVIC), each lasting for $5 \mathrm{~s}$. Three minutes were allowed between trials. To assess RFD, participants were instructed to apply "as much force as possible, as fast as possible" and were verbally encouraged throughout the contraction to ensure maximal effort. $F$ and RFD were evaluated in both the right and left legs at time intervals $0-50 \mathrm{~ms}\left(\mathrm{~F}_{0-50} ; R_{F} D_{\text {early }}\right), 0-200\left(\mathrm{~F}_{0-200} ; \mathrm{RFD}_{\text {late }}\right)$, Peak $\left(R F D_{\text {max }}\right)$, and time-to-peak ( $\left.R F D_{\text {time-to-peak }}\right)$. The right leg was always evaluated first.

\section{Balance test}

Static balance was performed with the participant standing quietly over a portable force platform (Kistlet 9286BA; Kistler Group, Winterthur, Switzerland), barefoot, with the legs shoulder-width apart and arms hanging at the sides. Each participant completed a 60 -s trial with the eyes open and another 60-s trial with the eyes closed, before and after each training session. The mean anterior/ posterior displacement (MAPD), mean medial/lateral displacement (MMLD), total sway displacement (TSD), and sway area (SA) were determined. The variables were calculated using the following formulas [39]:
$M A P D=\frac{\sum A B S\left(Y_{i}-\frac{\sum\left(Y_{i}\right)}{N}\right)}{N}$

$M M L D=\frac{\sum A B S\left(X_{i}-\frac{\sum\left(X_{i}\right)}{N}\right)}{N}$

$\mathrm{TSC}=\sum \sqrt{\left(\mathrm{Y}_{i+1}-\mathrm{Y}_{\mathrm{i}}\right)^{2}+\left(\mathrm{X}_{i+1}-\mathrm{X}_{\mathrm{i}}\right)^{2}}$

\section{Sit-to-stand test}

The sit-to-stand test measured the time it took to complete one repetition of standing as quickly as possible from the sitting position. The participants sat in a chair, with the arms crossed over their chest, back straight, knees flexed at $90^{\circ}$, and with both feet in full contact with the floor. The height of the chair was adjusted based on the dimensions of the patient's lower limb. The test was terminated when the trunk and knees were fully extended. Participants performed two trials, where the best trial was used for analysis. The speed at which patients performed the test was measured with the Sit-to-Stand app [40].

\section{Statistical analyses}

Data collection, treatment, and analysis were performed using IBM SPSS for Statistics for Windows (version 20.0; IBM Corp., Armonk, NY, USA). Descriptive statistics (mean \pm SD) were calculated. Before using parametric tests, the assumption of normality and homoscedasticity was confirmed with the Shapiro-Wilks test. Student's t-test for paired samples was used to test for significant changes between pre- and post-training for each group, separately. Analysis of covariance (ANCOVA) was performed with the EDSS score as a covariate to evaluate the differences between hypoxia and normoxia sessions. For all procedures, the statistical significance was set at $\mathrm{p} \leq 0.05$.

\section{Results}

Overall, 13 pwMS volunteered to participate in this clinical trial. Three participants dropped out of the study for different reasons (injury outside of the study intervention and incompatibility with the visiting hours during the study). Thus, a total of 10 participants with MS completed the study ( $30 \%$ males, $44.4 \pm 7.7$ years old, $164.3 \pm 8.9 \mathrm{~cm}$ tall, $65.2 \pm 11.1 \mathrm{~kg}$ of body mass, $2.5 \pm 1.3$ Expanded Disability Status Scale, and with a body mass index of $24.1 \pm 4.0 \mathrm{~kg} \cdot \mathrm{m}^{-2}$ ), including all testing assessments (• Fig. 1S). None of the participants had adverse effects. Also, no training had to be interrupted owing to a high rating of perceived exertion.

During the hypoxic session, pwMS showed a peripheral oxygen saturation of $92.50 \pm 0.577$ (before set 1 ), $92.25 \pm 1.50$ (before set 6 ) and $92.00 \pm 1.63$ (after set 12). During the normoxic session, peripheral oxygen saturation was $98.17 \pm 1.17$ (before set 1), $98.00 \pm 0.63$ (before set 6 ) and $97.67 \pm 1.21$ (after set 12). Differences were found in all time points in peripheral oxygen saturation $(p \leq 0.05)$.

As for the $\mathrm{SMO}_{2}$ in the gastrocnemius lateralis, pwMS had $74.39 \pm 11.77 \%$ and $78.06 \pm 8.43 \%$ in the right leg and $63.82 \pm 19.91 \%$ and $79.27 \pm 15.54 \%$ in left leg in hypoxic and nor- 


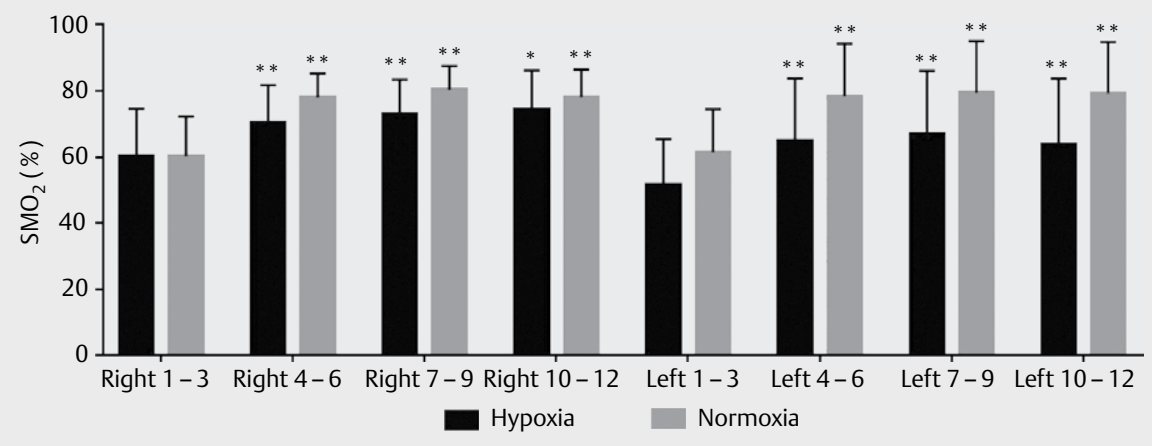

- Fig. 1 Effects of a whole-body vibration training session on muscle oxygen saturation.

- Table 1 Comparison between hypoxia and normoxia and effect of EDSS in neuromuscular outcomes.

\begin{tabular}{|c|c|c|c|c|c|c|}
\hline \multirow{3}{*}{ Neuromuscular outcomes (Hyp vs. Norm) } & \multicolumn{6}{|c|}{ ANCOVA interactions $\left(F, p, E S \eta^{2}\right)$} \\
\hline & \multicolumn{3}{|c|}{ Conditioning Effect } & \multicolumn{3}{|c|}{ EDSS Effect } \\
\hline & $\mathbf{F}$ & p & $E S \eta^{2}$ & $F$ & $\mathbf{P}$ & $E S \eta^{2}$ \\
\hline$F_{0-50}$ Right $(N \cdot m)$ & 0.165 & 0.690 & 0.010 & 0.006 & 0.941 & 0.000 \\
\hline$F_{0-50} \operatorname{Left}(\mathrm{N} \cdot \mathrm{m}$ & 0.291 & 0.596 & 0.016 & 0.404 & 0.534 & 0.023 \\
\hline$F_{0-200}$ Right $(N \cdot m)$ & 0.238 & 0.632 & 0.014 & 0.305 & 0.588 & 0.017 \\
\hline$F_{0-200} \operatorname{Left}(N \cdot \mathrm{m})$ & 0.241 & 0.630 & 0.014 & 0.194 & 0.666 & 0.011 \\
\hline$R F D_{\text {early }} \operatorname{Right}\left(\mathrm{N} \cdot \mathrm{m} \cdot \mathrm{s}^{-1}\right)$ & 0.005 & 0.944 & 0.000 & 0.397 & 0.537 & 0.023 \\
\hline$R F D_{\text {early }}$ Left $\left(N \cdot m \cdot s^{-1}\right)$ & 0.005 & 0.942 & 0.000 & 0.295 & 0.594 & 0.017 \\
\hline $\operatorname{RFD}_{\text {late }}$ Right $\left(\mathrm{N} \cdot \mathrm{m} \cdot \mathrm{s}^{-1}\right)$ & 0.165 & 0.689 & 0.009 & 0.798 & 0.384 & 0.044 \\
\hline $\mathrm{RFD}_{\text {late }}$ Left $\left(\mathrm{N} \cdot \mathrm{m} \cdot \mathrm{s}^{-1}\right)$ & 0.496 & 0.491 & 0.028 & 0.204 & 0.657 & 0.012 \\
\hline $\operatorname{RFD}_{\max }$ Right $\left(\mathrm{N} \cdot \mathrm{m} \cdot \mathrm{s}^{-1}\right)$ & 1.052 & 0.319 & 0.057 & 0.330 & 0.573 & 0.018 \\
\hline$R F D_{\max } \operatorname{Left}\left(\mathrm{N} \cdot \mathrm{m} \cdot \mathrm{s}^{-1}\right)$ & 0.782 & 0.389 & 0.043 & 0.243 & 0.628 & 0.013 \\
\hline$R F D_{\text {timetopeak }}$ Right (s) & 0.071 & 0.793 & 0.004 & 0.271 & 0.609 & 0.016 \\
\hline$R F D_{\text {timetopeak }}$ Left (s) & 0.184 & 0.674 & 0.010 & 1.673 & 0.213 & 0.089 \\
\hline
\end{tabular}

moxic conditions, respectively, after the final interval of the session

( $\triangleright$ Fig. 1).

Statistically significant pre-post differences were shown in $\mathrm{SMO}_{2}$ from the initial interval of the session to the next intervals of the session in both hypoxia and normoxia (see in > Table 1S).

No significant pre-post differences were found in $\mathrm{F}_{0-50}, \mathrm{~F}_{0-200}$,

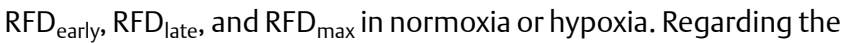
$\mathrm{RFD}_{\text {timetopeak }}$, there was a significant pre-post increase in hypoxia in the left leg $(t=2.66, p=0.02)$ and a tendency for an increase in the right leg $(t=2.13, p=0.06)$. No statistically significant differences were found in this variable under the normoxic condition (see Table 2S).

Regarding the sit-to-stand test, no pre-post differences were found. TSD, MMLD, MAPD, and SA with eyes open and closed did not show any statistically significant pre-post changes in both normoxia and hypoxia (see $>$ Table 3S).

No significant differences between normoxia and hypoxia were observed in Right ${ }_{1-6} \mathrm{SMO}_{2}\left(\mathrm{~F}=1.901\right.$, ES = 0.105), Right ${ }_{1-9} \mathrm{SMO}_{2}$ $(F=1.052, E S=0.061)$, Right ${ }_{1-12} S_{M O}(F=0.402, E S=0.024)$, Left $_{1-6} \mathrm{SMO}_{2}(F=0.057, \mathrm{ES}=0.003)$, Left ${ }_{1-9} \mathrm{SMO}_{2}(F=0.010$,
$\mathrm{ES}=0.001)$, Left $1-12 \mathrm{SMO}_{2}(\mathrm{~F}=0.252, \mathrm{ES}=0.014)$, or Sit-to-Stand $(F=3.184, E S=0.150)$. No differences between normoxia and hypoxia were observed in balance variables (see $>$ Table 4 S).

No significant differences between normoxia and hypoxia were observed in F or RFD. - Table 1 shows the comparison between condition and effect of EDSS on neuromuscular outcomes.

\section{Discussion}

The present study demonstrated that neuromuscular performance or functional capacity immediately after a single session of WBVT does not decline in pwMS in either hypoxic and normoxic condi-

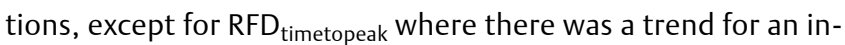
crease in the right leg and a significant increase in left leg in hypoxia. In addition, muscle oxygen saturation of the gastrocnemius lateralis increased during the session under both conditions.

\section{Muscle oxygen saturation}

The $\mathrm{SMO}_{2}$ levels measured in the gastrocnemius lateralis of the pwMS increased during the WBVT session compared to the initial 
values. This is in accordance with a meta-analysis that studied the effect of a WBVT session on muscle oxygenation in different muscles and showed increases in the gastrocnemius lateralis and not in other muscles, such as the rectus femoris or vastus lateralis [41]. In addition, the aforementioned meta-analysis indicated that frequency affects $\mathrm{SMO}_{2}$. Lower frequencies $(5-25 \mathrm{~Hz})$ have demonstrated a greater increase in $\mathrm{SMO}_{2}$, as the rate of muscle contraction allows for more perfusion time between contractions compared to higher vibration frequencies [41]. However, this study used a higher frequency $(35 \mathrm{~Hz})$, as used in other studies in populations with reduced mobility [42] and still demonstrated a significant increase in $\mathrm{SMO}_{2}$ during WBVT. Because skeletal muscle oxidative metabolism and blood flow are related to an increase in oxygen demand, the observed increase in $\mathrm{SMO}_{2}$ suggests that there was an increase in muscle activities [43], which could in turn lead to muscle adaptations derived from long-term training programs. Furthermore, individualized prescribed vibration frequency could lead to greater optimization and, therefore, greater neuromuscular adaptation.

Studies of hypoxic resistance training have shown that lower oxygen exposure could improve muscle hypertrophic responses via myogenesis and immune cell-dependent muscle regeneration [35]. The acute effect of hypoxia on $\mathrm{SMO}_{2}$ seems to be similar to normoxic condition at low training intensities, producing a plateau at high intensities in the hypoxic condition [44].

\section{Neuromuscular performance}

To our knowledge, only two studies has previously analyzed the acute effects of WBVT on muscle performance in this population: Jackson et al. [45], who studied isometric strength in knee flexion and extension after a 30-second exposure; and Andreu et al. [24], who investigated MVIC after a WBVT session. In accordance with the present results, these researchers found no changes after a training session compared to baseline values. In the present study, both RFD early and RFD late were not altered, which suggests that pwMS did not present any central fatigue (i. e., RFD early), or periph-

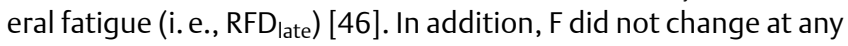
of the time intervals, which supports the aforementioned findings.

RFD is closely related with the activities of daily living [47], balance control [48], and the risk of falls [49]. Functional performance in activities of daily living [50], balance control and falls [51] are main problems for pwMS. In this population, it is important that acute fatigue generated after an exercise training session is not high in order to avoid a decrease in their ability to produce explosive force that could lead to a fall or a sudden decrease in their functional autonomy (inability to get up from a chair, inability to climb stairs, etc.) [52]. Therefore, WBVT is shown to be a safe method for pwMS, as improvements in muscle performance after several weeks of training have been demonstrated [14], and the acute effects of this type of training are shown to be safe $[24,45,53]$. Moreover, the increases in $\mathrm{SMO}_{2}$ show that muscular effort is occurring with very low neuromuscular fatigue.

For RFD $_{\text {late }}$ and $\mathrm{F}_{0-200}$, no changes were found after training with respect to baseline values in either hypoxia and normoxia. These results are in agreement with the findings by Andreu et al. [24] that showed no change in the central activation ratio after a WBVT session. $\mathrm{RFD}_{\text {late }}$ and $\mathrm{F}$ are related to the structural components of force, mainly with muscle size [46]. As with RFD early, an acute decrease in RFD $_{\text {late }}$ could be a problem for pwMS, especially in tasks such as walking [54]. In hypoxia, RFD $_{\text {timetopeak }}$ tends to increase in the right leg and increases significantly in the left leg. These results are consistent with Taylor et al. [55] that greater recruitment and higher rate coding in hypoxia with respect to normoxia in order to maintain the same intensity of exercise. Any increase in the time to reach maximum RFD would suggest greater central fatigue. Oliveira et al. [56] stated that RFD early is closely related to the mechanisms of the central nervous system and occurs between $0-100 \mathrm{~ms}$. Thus, $\mathrm{RFD}_{\text {timetopeak }}$ would be related to RFD early, and would be affected by central fatigue, which was observed under the hypoxic condition.

There were no significant differences in neuromuscular variables between hypoxia and normoxia. To our knowledge, this is the first study to evaluate the acute effects after a vibratory training session in hypoxia. Some studies have investigated the acute effects of strength under hypoxic conditions. Some authors have found increases in lactate concentration, decreases in $\mathrm{SMO}_{2}, \mathrm{pH}$, and muscle performance compared to the normoxia condition following strength training session $[32,57]$. However, in agreement with other authors, our results showed that physical performance, $\mathrm{SMO}_{2}$, and surface electromyography is no different between resistance exercise under normoxic or hypoxic condition $[33,58]$. In addition, a meta-analysis by Ramos-Campo et al. [27] that analyzed the effectiveness of long-term hypoxic training on muscle strength concluded that there was no evidence that hypoxia favors improvement in muscle strength over normoxic training. Inness et al. [59] found enhancements in strength in the resistance group with hypoxia despite no significant changes in lean mass, which could indicate a higher activation of the neural component during a strength session under hypoxia.

\section{Functional capacity}

Freitas et al. [53] studied the acute effects of WBVT on balance and mobility after a session in women with MS. The authors used 5 series of 1-minute vibration, with 1 min of rest between intervals, and a frequency of $30 \mathrm{~Hz}$ and amplitude of $3 \mathrm{~mm}$ (parameters similar to those used in this paper). In accordance with the present results, the authors found no pre-post changes in mobility and balance, measured with Timed Up and Go Test, the 500 m walk and limits of stability, respectively. In a recent study, Andreu et al. [24] also concluded that a WBVT session did not modify the mobility in pwMS. Although the acute effects of this type of training protocol did not appear to have a strong impact on balance and functional ability of pwMS [24, 45, 53], the potential long-term benefits of WBVT could improve gait [13]; reduce fall risk [21]; and improve mobility, balance, and postural control [60].

Regarding balance control, it has been shown that pwMS have a greater medial/lateral displacement when standing compared to the healthy, control group [61-63] Other studies have demonstrated that pwMS have less displacement of the center of pressure during static and dynamic tasks when compared to non-MS individuals and that this could serve as an adaptive strategy for pwMS to overcome the deficiency in postural control that could result in a fall [64-65]

A previous study by Morrison et al. [66] concluded that MMLD is the variable most related to the risk of falls, so it is important that 
this variable is not affected after training. An increase in MMLD could lead to an increased risk of falls. This study found no pre-post changes in any balance variable when eyes were closed and open. Previous research has shown that pwMS, in addition to having poorer balance due to damage to the neuromuscular component, also present a poor vestibular, visual, and proprioceptive system $[63,67,68]$. However, the dependence of the three systems is great in pwMS in order to maintain vertical balance [66]. Thus, the absence of fatigue after a WBVT session is essential because it does not increase the risk of falls in this population as neither strength nor balance are decreased. Our study also has certain limitations. The main limitation is the low sample size. In addition, another possible limitation was the non-individualization of the WBVT training.

This study provides important information about how a single WBVT session affects neuromuscular performance, balance, mobility, and $\mathrm{SMO}_{2}$ in pwMS. One of the more significant findings to emerge from this research is that vibration training requires muscle demand during the session without causing significant amounts of fatigue to the neuromuscular system.

\section{Acknowledgements}

We are grateful to all the participants in this study.

\section{Conflict of Interest}

The authors declare that they have no conflict of interest.

\section{References}

[1] Barten LJ, Allington DR, Procacci KA et al. New approaches in the management of multiple sclerosis. Drug Des Devel Ther 2010; 4: 343-366

[2] Browne P, Chandraratna D, Angood C et al. Atlas of multiple sclerosis 2013: A growing global problem with widespread inequity. Neurology 2014; 83: 1022-1024

[3] Martin CL, Phillips BA, Kilpatrick T] et al. Gait and balance impairment in early multiple sclerosis in the absence of clinical disability. Mult Scler 2006; 12: 620-628

[4] Jørgensen MLK, Dalgas U, Wens I et al. Muscle strength and power in persons with multiple sclerosis - a systematic review and meta-analysis. J Neurol Sci 2017; 376: 225-241

[5] Thoumie P, Lamotte D, Cantalloube $S$ et al. Motor determinants of gait in 100 ambulatory patients with multiple sclerosis. Mult Scler 2005; 11: $485-491$

[6] de Silva R, Greenfield J, Cook A et al. Guidelines on the diagnosis and management of the progressive ataxias. Orphanet J Rare Dis 2019; 14 : $1-10$

[7] Motl RW. Ambulation and multiple sclerosis. Phys Med Rehabil Clin N Am 2013; 24: 325-336

[8] Weideman AM, Tapia-Maltos MA, Johnson K et al. Meta-analysis of the age-dependent efficacy of multiple sclerosis treatments. Front Neurol 2017; 8: 577

[9] Amatya B, Khan F, Galea M. Rehabilitation for people with multiple sclerosis: An overview of Cochrane Reviews. Cochrane Database Syst Rev 2019; 1: CD012732
[10] Tae-Woon K, Yun-Hee S. Regular exercise promotes memory function and enhances hippocampal neuroplasticity in experimental autoimmune encephalomyelitis mice. Neuroscience 2017; 346: 173-181

[11] White L], Castellano V. Exercise and brain health - implications for multiple sclerosis. Sports Med 2008; 38: 91-100

[12] Motl RW, Pilutti LA. The benefits of exercise training in multiple sclerosis. Nat Rev Neurol 2012; 8: 487-497

[13] Kantele S, Karinkanta S, Sievänen H. Effects of long-term whole-body vibration training on mobility in patients with multiple sclerosis: $\mathrm{A}$ meta-analysis of randomized controlled trials. J Neurol Sci 2015; 358: $31-37$

[14] Castillo-Bueno I, Ramos-Campo DJ, Rubio-Arias JA. Effects of whole-body vibration training in patients with multiple sclerosis: A systematic review. Neurologia 2018; 33: 534-548

[15] Krause A, Gollhofer A, Freyler K et al. Acute corticospinal and spinal modulation after whole body vibration. J Musculoskelet Neuronal Interact 2016; 16: 327-338

[16] Baudry S, Duchateau J. Independent modulation of corticospinal and group I afferents pathways during upright standing. Neuroscience 2014; 275: 162-169

[17] Hodapp M, Vry J, Mall V et al. Changes in soleus H-reflex modulation after treadmill training in children with cerebral palsy. Brain 2009; 132: 37-44

[18] Hoque M, Borich M, Sabatier M et al. Effects of downslope walking on Soleus $\mathrm{H}$-reflexes and walking function in individuals with multiple sclerosis: A preliminary study. NeuroRehabilitation 2019; 44: 587-597

[19] Maeda N, Urabe Y, Sasadai J et al. Effect of whole-body-vibration training on trunk-muscle strength and physical performance in healthy adults: Preliminary results of a randomized controlled trial. J Sport Rehabil 2016; 25: 357-363

[20] Schuhfried O, Mittermaier C, Jovanovic T et al. Effects of whole-body vibration in patients with multiple sclerosis: A pilot study. Clin Rehabil 2005; 19: 834-842

[21] Yang F, Finlayson M, Bethoux F et al. Effects of controlled whole-body vibration training in improving fall risk factors among individuals with multiple sclerosis: A pilot study. Disabil Rehabil 2018; 40: 553-560

[22] Wunderer K, Schabrun SM, Chipchase LS. Effects of whole body vibration on strength and functional mobility in multiple sclerosis. Physiother Theory Pract 2010; 26: 374-384

[23] Mason RR, Cochrane DJ, Denny G] et al. Is 8 weeks of side-alternating whole-body vibration a safe and acceptable modality to improve functional performance in multiple sclerosis? Disabil Rehabil 2012; 34 : 647-654

[24] Andreu L, Ramos-Campo D], Ávila-Gandía V et al. Acute effects of whole-body vibration training on neuromuscular performance and mobility in hypoxia and normoxia in persons with multiple sclerosis: a crossover study. Mult Scler Relat Disord 2020; 37: 101454

[25] Hilgers C, Mündermann A, Riehle $\mathrm{H}$ et al. Effects of whole-body vibration training on physical function in patients with multiple sclerosis. NeuroRehabilitation 2013; 32: 655-663

[26] Cochrane DJ, Stannard SR, Firth EC et al. Acute whole-body vibration elicits post-activation potentiation. Eur J Appl Physiol 2010; 108: 311-319

[27] Ramos-Campo D], Scott BR, Alcaraz PE et al. The efficacy of resistance training in hypoxia to enhance strength and muscle growth: $\mathrm{A}$ systematic review and meta-analysis. Eur J Sport Sci 2018; 18: 92-103

[28] Scott BR, Slattery KM, Sculley DV et al. Hypoxia and resistance exercise: A comparison of localized and systemic methods. Sports Med 2014; 44: 1037-1054

[29] Lundby C, Calbet JAL, Robach P. The response of human skeletal muscle tissue to hypoxia. Cell Mol Life Sci 2009; 66: 3615-3623 
[30] Zoll ], Ponsot E, Dufour S et al. Exercise training in normobaric hypoxia in endurance runners. III. Muscular adjustments of selected gene transcripts. J Appl Physiol (1985) 2006; 100: 1258-1266

[31] Casey DP, Joyner MJ. Compensatory vasodilatation during hypoxic exercise: Mechanisms responsible for matching oxygen supply to demand. J Physiol 2012; 590: 6321-6326

[32] Ramos-Campo DJ, Rubio-Arias JA, Dufour $S$ et al. Biochemical responses and physical performance during high-intensity resistance circuit training in hypoxia and normoxia. Eur J Appl Physiol 2017; 117: 809-818

[33] Scott BR, Slattery KM, Sculley DV et al. Acute physiological responses to moderate-load resistance exercise in hypoxia. J Strength Cond Res 2017; 31: 1973-1981

[34] Kon M, Ohiwa N, Honda A et al. Effects of systemic hypoxia on human muscular adaptations to resistance exercise training. Physiol Rep 2014; 2: e12033

[35] Britto FA, Gnimassou O, De Groote E et al. Acute environmental hypoxia potentiates satellite cell-dependent myogenesis in response to resistance exercise through the inflammation pathway in human. FASEB J 2019; 34: 1-16

[36] World Medical Association. Declaration of Helsinki - Ethical principles for medical research involving human subjects (2000). Bull World Heal Organ 2001; 79: 373-374

[37] Harriss DJ, Macsween A, Atkinson G. Ethical standards in sport and exercise science research: 2020 update. Int J Sports Med 2019; 40: 813-817

[38] Thompson AJ, Banwell BL, Barkhof F et al. Diagnosis of multiple sclerosis: 2017 revisions of the McDonald criteria. Lancet Neurol 2018; 17: $162-173$

[39] Kantner RM, Rubin AM, Armstrong CW et al. Stabilometry in balance assessment of dizzy and normal subjects. Am J Otolaryngol 1991; 12: 196-204

[40] Ruiz-Cárdenas JD, Rodríguez-Juan JJ, Smart RR et al. Validity and reliability of an iPhone App to assess time, velocity and leg power during a sit-to-stand functional performance test. Gait Posture 2018; 59: 261-266

[41] Games KE, Sefton JEM, Wilson AE. Whole-body vibration and blood flow and muscle oxygenation: A meta-analysis. J Athl Train 2015; 50: 542-549

[42] Yarar-Fisher C, Pascoe DD, Gladden LB et al. Acute physiological effects of whole body vibration (WBV) on central hemodynamics, muscle oxygenation and oxygen consumption in individuals with chronic spinal cord injury. Disabil Rehabil 2014; 36: 136-145

[43] Neary JP. Application of near infrared spectroscopy to exercise sports science. Can J Appl Physiol 2004; 29: 488-503

[44] Subudhi AW, Dimmen AC, Roach RC. Effects of acute hypoxia on cerebral and muscle oxygenation during incremental exercise. J Appl Physiol (1985) 2007; 103: 177-183

[45] Jackson KJ, Merriman HL, Vanderburgh PM et al. Acute effects of whole-body vibration on lower extremity muscle performance in persons with multiple sclerosis. J Neurol Phys Ther 2008; 32: 171-176

[46] Maffiuletti NA, Aagaard P, Blazevich AJ et al. Rate of force development: physiological and methodological considerations. Eur J Appl Physiol 2016; 116: 1091-1116

[47] Aagaard P, Suetta C, Caserotti P et al. Role of the nervous system in sarcopenia and muscle atrophy with aging: Strength training as a countermeasure. Scand J Med Sci Sport 2010; 20: 49-64

[48] Hess JA, Woollacott M, Shivitz N. Ankle force and rate of force production increase following high intensity strength training in frail older adults. Aging Clin Exp Res 2006; 18: 107-115

[49] Rubenstein LZ. Falls in older people: epidemiology, risk factors and strategies for prevention. Age Ageing 2006; 35: 37-41
[50] Buzaid A, Dodge MP, Handmacher L et al. Activities of daily living. Evaluation and treatment in persons with multiple sclerosis. Phys Med Rehabil Clin N Am 2013; 24: 629-638

[51] Gunn H, Markevics S, Haas B et al. Systematic review: The effectiveness of interventions to reduce falls and improve balance in adults with multiple sclerosis. Arch Phys Med Rehabil 2015; 96: 1898-1912

[52] Cruickshank TM, Reyes AR, Ziman MR. A systematic review and meta-analysis of strength training in individuals with multiple sclerosis or Parkinson disease. Medicine (Baltimore) 2015; 94: e411

[53] Freitas EDS, Frederiksen C, Miller RM et al. Acute and chronic effects of whole-body vibration on balance, postural stability, and mobility in women with multiple sclerosis. Dose-Response 2018; 16: 1-13

[54] Kjølhede T, Vissing K, Langeskov-Christensen D et al. Relationship between muscle strength parameters and functional capacity in persons with mild to moderate degree multiple sclerosis. Mult Scler Relat Disord 2015; 4: 151-158

[55] Taylor AD, Bronks R, Smith P et al. Myoelectric evidence of peripheral muscle fatigue during exercise in severe hypoxia: Some references to $\mathrm{m}$. vastus lateralis myosin heavy chain composition. Eur J Appl Physiol Occup Physiol 1997; 75: 151-159

[56] de Oliveira FBD, Rizatto GF, Denadai BS. Are early and late rate of force development differently influenced by fast-velocity resistance training? Clin Physiol Funct Imaging 2013; 33: 282-287

[57] Ramos-Campo D], Rubio-Arias ], Freitas TT et al. Acute physiological and performance responses to high-intensity resistance circuit training in hypoxic and normoxic conditions. J Strength Cond Res 2017; 31: 1040-1047

[58] Scott BR, Slattery KM, Sculley DV et al. Physical performance during high-intensity resistance exercise in normoxic and hypoxic conditions. J Strength Cond Res 2015; 29: 807-815

[59] Mathew MW, Billaut F, Walker E] et al. Heavy resistance training in hypoxia enhances 1RM squat performance. Front Physiol 2016; 7: 502

[60] Alashram AR, Padua E, Annino G. Effects of whole body vibration on motor impairments in patients with neurological disorders. Am J Phys Med Rehabil 2019; 98: 1084-1098

[61] Hoang PD, Cameron MH, Gandevia SC et al. Neuropsychological, balance, and mobility risk factors for falls in people with multiple sclerosis: a prospective cohort study. Arch Phys Med Rehabil 2014; 95: 480-486

[62] Sosnoff J], Shin S, Motl RW. Multiple sclerosis and postural control: the role of spasticity. Arch Phys Med Rehabil 2010; 91: 93-99

[63] Kanekar N, Lee Y], Aruin AS. Frequency analysis approach to study balance control in individuals with multiple sclerosis. J Neurosci Methods 2014; 222: 91-96

[64] Karst GM, Venema DM, Roehrs TG et al. Center of pressure measures during standing tasks in minimally impaired persons with multiple sclerosis. J Neurol Phys Ther 2005; 29: 170-180

[65] Van Emmerik REA, Remelius JG, Johnson MB et al. Postural control in women with multiple sclerosis: effects of task, vision and symptomatic fatigue. Gait Posture 2010; 32: 608-614

[66] Morrison S, Rynders CA, Sosnoff J]. Deficits in medio-lateral balance control and the implications for falls in individuals with multiple sclerosis. Gait Posture 2016; 49: 148-154

[67] Herrera WG. Vestibular and other balance disorders in multiple sclerosis. Differential diagnosis of disequilibrium and topognostic localization. Neurol Clin 1990; 8: 407-420

[68] Nelson SR, di Fabio RP, Anderson JH. Vestibular and sensory interaction deficits assessed by dynamic platform posturography in patients with multiple sclerosis. Ann Otol Rhinol Laryngol 1995; 104: 62-68 